\title{
A strategy to obtain axenic cultures of Arthrospira spp. cyanobacteria
}

\author{
Lucia Sena $\cdot$ Diego Rojas $\cdot$ Edie Montiel $\cdot$ \\ Héctor González · Josnell Moret • Leopoldo Naranjo
}

Received: 31 May 2009/Accepted: 20 August 2010/Published online: 1 September 2010

(C) The Author(s) 2010. This article is published with open access at Springerlink.com

\begin{abstract}
A strategy to obtain axenic cultures of the cyanobacterium Arthrospira sp. ('platensis') Lefevre 1963/ M-132-1 strain, consisting of a series of physical and chemical procedures, and the application of an optimized pool of antibiotics, is described in this paper. This strategy, which is an inexpensive and fast way to obtain axenic cultures, can be applied to Arthrospira spp. from culture collections or samples from their natural habitats to eliminate a wide spectrum of contaminants. A high alkaline treatment ( $\mathrm{pH} \mathrm{12,} \mathrm{using} \mathrm{KOH})$ of $72 \mathrm{~h}$ is a determinant initial procedure applied to eliminate protozoa and Microcystis sp. Bacteria were eliminated by an optimal antibiotic pool treatment, and Chroococcus sp. residuals were discarded by serial dilution. Optimal concentrations of the antibiotics composing the pool were obtained by a $2^{4}$ factorial central composite rotatable design (CCRD) and Response Surface Methodology (RSM), resulting in: ampicillin $61.6 \mu \mathrm{g} / \mathrm{ml}$, penicillin $85.8 \mu \mathrm{g} / \mathrm{ml}$, cefoxitin $76.9 \mu \mathrm{g} / \mathrm{ml}$, and meropenem $38.9 \mu \mathrm{g} / \mathrm{ml}$. The results also indicate that cefoxitin was the most effective antibiotic of this pool. After obtaining the axenic culture, identification of Lefevre 1963/M-132-1 strain was performed using amplification and sequencing of the ITS region (including part of 16S rRNA, tRNA Ile, ITS, tRNA Ala and part of $23 \mathrm{~S}$ rRNA region) and fatty acid composition data. Data
\end{abstract}

L. Sena · D. Rojas · J. Moret · L. Naranjo ( $\square)$

Dirección de Área de Energía y Ambiente, Fundación Instituto de Estudios Avanzados (IDEA), Sartenejas, Caracas 1080, Venezuela

e-mail: lnaranjo@idea.gob.ve

E. Montiel · H. González

Grupo de Optimización Agrícola (GOA), Instituto de Zoología y

Ecología Tropical (IZET), Facultad de Ciencias, Universidad

Central de Venezuela (UCV), Caracas, Venezuela base comparison revealed that Lefevre strain is closely related to A. platensis species (99\% identity), while fatty acid composition data suggested A. maxima. These seemingly contradictory results are discussed.

Keywords Antibiotics - Axenic cultures - Central composite rotatable design (CCRD) - Optimization · Response surface methodology (RSM) - Arthrospira

\section{Introduction}

Arthrospira sp. ('platensis') is a filamentous, photoautotrophic, alkaliphilic cyanobacterium that belongs to the Family Oscillatoriaceae, Division Cyanophyta. This cyanobacterium blooms in bicarbonate-rich environments (Ciferri 1983), and has gained a significant position in recent years as a source of proteins and pigments in the food, pharmaceutical, and cosmetic industries (Ciferri and Tiboni 1985; Vonshak 1997; Mosulishvili et al. 2002).

Axenic cultures are important in genetic, biochemical, physiological and taxonomic studies. However, the use of Arthrospira spp. in biochemical and genetic studies has been limited due to the difficulties in obtaining axenic cultures (Vonshak 1997). Unialgal-free bacterial cultures are also valuable in the first step of scaling up Arthrospira (Spirulina) cultures, as undesired bacteria and cyanobacteria can grow in the same medium (Duerr et al. 1997). Worth mentioning is the example of Microcystis spp., which produces a potent hepatotoxin and potential carcinogen, called microcystin (Wang and Zhu 1996). Diverse methods of cyanobacteria purification have been described, and some of them may involve one or more steps, ranging from physical separation techniques to chemical or biochemical treatments. Some common methods are: plating 
procedures, growing in selective media, filtration, differential centrifugation, sonication, vortexing, dilution culture, purification by micropipetting, variation of intensity light, antibiotic treatments, toxic chemicals (e.g. germicides), ultraviolet light, gamma irradiation, heating, and rinsing, among others (Wieringa 1968; Castenholz 1970; Ogawa and Terui 1970; Carmichael and Gorham 1974; Vaara et al. 1979; Rippka 1988; Ferris and Hirsch 1991; Nola et al. 1992; Suresh et al. 1994; Kim et al. 1999; Andersen 2005; Gang-Guk et al. 2007).

There is not a single "best" purification method. Each one needs to be tailored according to the morphological and physiological properties of the contaminant and target species. Therefore, we must visualize the purification method as a "strategy" (a logic sequence of treatments that takes into account the specific properties of the contaminants and target species). Once a logical sequence is established, each step needs to be optimized.

This work presents a purification strategy composed of four optimized processing steps: (1) washing by filtration, (2) $\mathrm{pH}$ treatment, (3) antibiotic treatment and (4) serial dilution. These steps constitute an efficient and inexpensive methodology to obtain axenic cultures of Arthrospira spp. Other methods to obtain axenic cultures of Arthrospira spp. have been proposed (Ogawa and Terui 1970; Suresh et al. 1994; Gang-Guk et al. 2007), but they do not include a $\mathrm{pH}$ treatment followed by an optimized antibiotic treatment.

Although taxonomy is not the main focus of the present work, once the purification process was completed, it seemed logical to verify the identity of the strain, because there could be uncertainty about the identity of the purified strain. As was mentioned, one of the most common uses of axenic cultures is in taxonomic identification, but this use can be limited by multiple factors that impede implementation of a conventional method or technique. So it is necessary to devize a process that allows the efficient use of available techniques and data. In order to do this, the first step consists in reviewing the results obtained by other authors and evaluating the available palette of possibilities. This guides us in the building of the logical steps to identify the axenic strain obtained. In our case, the Lefevre strain underwent a morphological change (from helical to straight) and a taxonomic reclassification, which makes the identification process more difficult.

Despite A. platensis and A. maxima having been separated into two different taxonomic entities based on morphological characters and distribution, an intensive taxonomic debate is still taking place over the classification of these Arthrospira spp. (Hindak 1985; Komárek 2000; Komárek and Lund 1990; Tomaselli 1997). Strain Lefevre 1963/M-132-1 was originally identified as Arthrospira sp. ('platensis') and was later reclassified as Arthrospira maxima at CCALA under the designation given by Setchell et Gardner 1917.

Komárek and Lund (1990) recognize gas vacuoles as an important taxonomic character for the genus Arthrospira. In fact, Komárek (2000) distinguished in the genus Arthrospira (sensu stricto) two groups of species: benthic and periphytic species with no aerotopes in their cells (A. jenneri, A. platensis), and planktic species with gas vesicles in their cells, living in solitary trichomes (A. maxima, A. fusiformis). Mühling et al. (2006) argued against the use of this taxonomic character, because gas vacuole development in cultures may be markedly influenced by the laboratory environment. This disagreement led us to avoid the use of this taxonomic character.

Taxonomic identification of Arthrospira species is difficult because of the morphological variability under different environmental conditions. In fact, in our laboratory, Lefevre 1963/M-132-1 strain underwent a morphological shift from the usual helical shape to a linear one. The tendency to form straight trichomes is an important phenomenon that from the taxonomic point of view, has not yet been satisfactorily explained. This phenomenon had been considered for a long time as a permanent degeneration that could not be reversed (Komárek 2000; Komárek and Anagnostidis 2005; Tomaselli 1997). Nevertheless, Wang and Zhao (2005) described that under certain conditions linear filaments can revert to their original morphology.

Different analyses have been used to establish the genotypic diversity and phylogenetic relationship of Arthrospira strains (Viti et al. 1997; Scheldeman et al. 1999; Ballot et al. 2004; Baurain et al. 2002; Manen and Falque 2002; Mühling et al. 2006). Scheldeman et al. (1999) carried out an ARDRA (Amplified Ribosomal DNA Restriction Analysis) on the ITS of 37 cultivated clonal strains from four continents. Cluster analysis showed the separation of all the strains into two main clusters, with all strains closely related. Scheldeman et al. (1999) found no clear relationships between this division into two clusters and the geographic origin of the strains, or their designation in the culture collections, or their morphology.

Baurain et al. (2002) using amplification and sequencing of the whole ITS, showed a remarkable conservation of these sequences in 21 out of the 37 Arthrospira clonal strains isolated from the four continents, and grouped them into four different species (A. platensis, A. maxima, A. fusiformis, A. indica) in the culture collections (for a review on the adequate use of this tool in Cyanobacteria see Boyer et al. 2001). This same author found that the high conservation of ITS sequences is in contrast with the assignment to four different species, the great morphological variability of the strains, and their wide geographic distribution. This suggests that ITS sequences alone are 
insufficient to discriminate at this level. It is interesting to note that Baurain et al. (2002) also found that variants "straight" and "helical" that were isolated in clonal cultures presented identical ITS sequences (i.e., Arthrospira fusiformis, strain CCAP 1475/8). Indeed, when the dendrogram built by Scheldeman et al. (1999) is carefully examined it could be noted that the position of variants "straight" and "helical" is consistent with this fact, that is, grouped together inside the same genotypic cluster (e.g., Arthrospira sp. ('platensis') LAPORTE 1963/M132-2b and Arthrospira fusiformis, strain CCAP 1475/8). Those facts suggest that "straight" or "helical" morphologies do not affect the molecular taxonomy criteria used here.

Genuine Arthrospira strains have a significant proportion of $\gamma$-linolenic acid (GLA), no $\alpha$-linolenic acid (ALA), a low content of 16:1 fatty acids and a very low content of 16:2 fatty acids (Cohen and Vonshak 1991; Cohen et al. 1995). Mühling et al. (2005) studied the fatty acid composition for 35 Arthrospira strains, finding that the most abundant fatty acids were two polyunsaturated C18 acids, linoleic and $\gamma$-linolenic acid, and palmitic acid. They suggested that fatty acid composition can be used as an aid in differentiating between strains. It is important to note that straight morphotypes showed a similar fatty acid composition to that of the helical morphotypes of the same strains. This allowed the use of these data even if the strain underwent a morphological change. Additionally, Mühling et al. (2006) in order to investigate the extent to which it is possible to distinguish clades of strains within the genus Arthrospira, carried out an analysis of a wide range of phenotypic characters in 33 helical and 2 straight Arthrospira strains, and compare the results with those previously obtained by molecular techniques. This approach (in the "phenotypic characters") made a wide range of features (morphological, ultrastructural, physiological and biochemical), to converge, making it highly inclusive and comprehensive.

In Mühling's et al. (2006) work, multivariate analysis using the simple matching coefficient resolved the 33 helical strains into two clusters (one related with $A$. maxima, A. fusiformis and A. indica and one with most strains designated $A$. platensis). It is remarkable that Mühling et al. (2006) found that phenotypic clusters showed a high correlation with the characters describing the helical trichome shape, and also to the molecular clusters of the same strains reported in the literature. Establishing this is important because it implies some level of redundancy that allows the use of morphological and molecular data depending on their availability (in the problem at hand the fact that our strain has become straight, leaving virtually no major morphological criteria available). It is important to note that due to the loss of too many features (i.e., "morphological characters" and "character states") Mühling's numerical analysis did not include strains with straight trichomes.

In the case that concerns us, any sequence of logical steps to clarify the identity of the strain obtained, must take into account that no correlation between characters that describe the shape of helical trichomes can be drawn, due to the strain having become straight. Instead, molecular and biochemical features should be taken into account.

Tomaselli (1997) has indicated that molecular analysis is the best way to develop an improved classification system for Arthrospira species. However, for the above reasons, we believe that the molecular approach is not sufficient and should be complemented with other approaches that can discern the true identity of the axenic strain obtained. Moreover, the use of a single approach, either of molecular, biochemical, physiological or morphological nature, could lead to erroneous or contradictory results, as is exemplified in the results and discussion sections.

In this work, we report the results of the proposed strategy of purification and the molecular-biochemical identification of the Lefevre 1963/M-132-1 strain.

\section{Materials and methods}

\section{Strain and culture conditions}

Arthrospira sp. ('platensis') Lefevre 1963/M-132-1 strain (collected in Chad, Natron Lake), reclassified as Arthrospira maxima, was obtained from the Culture Collection of Phototrophic Organisms (CCALA) of the Czech Republic. The strain was grown under continuous fluorescent light at 1,200 lux (Light intensity meter x-101 Lutron, Taiwan) in AO (Aiba and Ogawa) culture medium (Aiba and Ogawa 1977) at $32^{\circ} \mathrm{C}$ and $150 \mathrm{rev} / \mathrm{min}$ (shaker incubator 311DS Labnet, USA).

Physical and chemical treatments

The first step of our strategy for purifying Arthrospira cultures was washing by filtration. Using a 47- $\mu \mathrm{m}$ pore membrane, $20 \mathrm{ml}$ of culture were washed with 11 of sterile carbonate and bicarbonate buffer. Filaments were then suspended in $40 \mathrm{ml}$ of AO medium; three suspensions of $40 \mathrm{ml}$ each were made to obtain a total of $120 \mathrm{ml}$ of washed culture.

The second step was $\mathrm{pH}$ treatment to eliminate Microcystis sp. Arthrospira cultures were subjected to four $\mathrm{pH}$ levels $(10,11,12$, and 13) during different periods of time (48, 72, and $96 \mathrm{~h}$ ) to determine the optimal $\mathrm{pH}$ treatment. Hence, 12 different treatments were defined, and two replicates were made for each one. In each flask, $50 \mathrm{ml}$ of 
micronutrients $\mathrm{AO}$ medium and $0.35 \mathrm{~g}$ of $\mathrm{NaHCO}_{3}$ were added; the $\mathrm{pH}$ was adjusted using a $5 \mathrm{M} \mathrm{KOH}$ solution, and subsequently inoculated with $5 \mathrm{ml}$ of Lefevre 1963/M-1321 strain culture (previously washed as mentioned above). Flasks were placed in the incubator shaker at $32^{\circ} \mathrm{C}$ and $150 \mathrm{rev} / \mathrm{min}$, under continuous fluorescent light. When the corresponding period of time elapsed, the $\mathrm{pH}$ was immediately reduced to a value between 9 and 9.6 by pouring in $50 \mathrm{ml}$ of carbonate and bicarbonate buffer. After that, samples from each $\mathrm{pH}$ treatment were microscopically examined for contaminant microorganisms.

The third step was a treatment with antibiotics to eliminate bacteria present in the culture. To determine the optimal concentrations of each antibiotic in the pool, a factorial experiment was carried out (CCRD and RSM, see the antibiotic experimental section below). The antibiotics selected to compose the pool were ampicillin, cefoxitin, penicillin G and meropenem. Successful Arthrospira cultures after $\mathrm{pH}$ treatment were subjected to thirty different combinations of antibiotics concentrations treatments and incubated in the dark, at $32^{\circ} \mathrm{C}$ and $150 \mathrm{rev} / \mathrm{min}$ for $48 \mathrm{~h}$. Cultures were then washed by centrifugation (centrifuge 5424 Eppendorf, Germany) at 14,000 rev/min for $10 \mathrm{~min}$; pellets were suspended in $3 \mathrm{ml}$ of AO medium and incubated at $200 \mathrm{rev} / \mathrm{min}$ for $1 \mathrm{~h}$. Finally, they were incubated at $32^{\circ} \mathrm{C}$ in a growth chamber until noticeable growth of Lefevre strain was observed ( 2 weeks).

The final step was serial dilution, which was used to eliminate the unicellular cyanobacterium Chroococcus sp. that remained after the antibiotic treatment. The dilutions used were 1:1,000, 1:10,000 and 1:100,000.

\section{Axenic culture verification}

The purity of Arthrospira cultures was determined before and after each step of the strategy by careful microscopic examination using phase contrast illumination (Carl Zeiss Axioskop 40, Germany). The efficacy of the treatments with antibiotics was assessed by inoculation $(0.1 \mathrm{ml}$ of treated culture) on AO agar (glucose $1 \%$, peptone $0.5 \%$, yeast extract $0.3 \%$ ). Bacteria FCU and filaments of Lefevre strain were counted; the latter in a Neubauer counting chamber (Hausser Brigth-Line, USA).

Antibiotic experimental design and statistical analysis

In order to obtain bacteria-free cultures of Arthrospira (after $\mathrm{pH}$ treatment), the optimal concentrations of the antibiotics that composed the pool were obtained by using Response Surface Methodology (RSM) (Montgomery 1997; Myers and Montgomery 2002). Ampicillin, cefoxitin, penicillin $G$ and meropenem were the antibiotics composing the pool, and their concentrations were the independent variables or factors. The experimental design was a Central Composite Rotatable Design (CCRD) completely balanced. CCRD involves outlining the concentration composition of the pool around a central combination, and subsequently used to develop the regression model. The design consists of a $2^{4}$ factorial (four-factor-twolevel pattern) with 30 runs, 16 factorial combination points, 6 centre points and 8 axial or star points (see Table 1). The intermediate level $(0)$ constitutes the centre point and the high $(+2)$ and low $(-2)$ levels constitute the axial or star points. Response variables were bacteria number (c.f.u.) and Lefevre strain filaments number. To find the relationship between the response and the independent variables, a second order model (regression equation) was used:

$Y=\beta_{0}+\sum_{i=1}^{k} \beta_{i} X_{i}+\sum_{i=1}^{k} \beta_{i i} X_{i}^{2}+\sum_{i<j}^{k} \sum \beta_{i j} X_{i} X_{j}+\varepsilon$.

where $X_{1}, X_{2}, X_{3} \ldots X_{k}$ are the independent variables which affect the response $\mathrm{Y}, \beta_{0}, \beta_{i}, \beta_{i i}$ and $\beta_{i j}(i=1-k$, $j=1-k$ ) are regression coefficients, and $\varepsilon$ is the random error. Analysis of variance was used to check the adequacy of the experimental design and optimization of the response variables. "SAS 9.1" and "Design Expert 7.1" software were used for the statistical analysis.

\section{Identification process}

A two-step identification process was designed, based on the literature reviewed (see Introduction) and the current characteristics of the Lefevre 1963/M-132-1 strain. In the first step we sequenced a 1,948 bp DNAfragment comprising the ITS region (including part of 16S rRNA, tRNA Ile, tRNA Ala and part of 23S rRNA region) and compared it with homologous DNA sequences deposited in GenBank database. The second step was determining the Lefevre 1963/M-132-1 strain fatty acid composition and comparing it with the data and criteria previously obtained by other authors. These two steps are described in detail below.

DNA extraction and molecular identification

For total DNA extraction, approximately $1.5 \mathrm{ml}$ cell suspension of the axenic Arthrospira culture was concentrated by centrifugation at $11,000 \mathrm{rev} / \mathrm{min}$ for $15 \mathrm{~min}$ (model 5429 Eppendorf, Germany). The pellet was stored at $-20^{\circ} \mathrm{C}$ overnight, and subsequently defrosted and centrifuged at 11,000 rev/min for $7 \mathrm{~min}$. DNA was extracted according to $\mathrm{Wu}$ et al. (2002). The molecular identification of Lefevre strain was carried out by PCR amplification and sequencing of the ITS region (including part of 16S rRNA, tRNA Ile, ITS, tRNA Ala and part of 23S rRNA region) 
Table 1 Experimental design (CCRD) with codes and variables used to optimise the concentration of antibiotics used to obtain bacteria-free cultures of Arthrospira sp. ('platensis')

\begin{tabular}{|c|c|c|c|c|c|c|c|c|c|}
\hline Position & $\begin{array}{l}\text { Run } \\
\text { no. }\end{array}$ & $\mathrm{X}_{1}$ & $\mathrm{X}_{2}$ & $\mathrm{X}_{3}$ & $\mathrm{X}_{4}$ & $\begin{array}{l}\text { Ampicillin } \\
(\mu \mathrm{g} / \mathrm{ml})\end{array}$ & $\begin{array}{l}\text { Cefoxitin } \\
(\mu \mathrm{g} / \mathrm{ml})\end{array}$ & $\begin{array}{l}\text { Penicillin } \\
(\mu \mathrm{g} / \mathrm{ml})\end{array}$ & $\begin{array}{l}\text { Meropenem } \\
(\mu \mathrm{g} / \mathrm{ml})\end{array}$ \\
\hline \multirow[t]{16}{*}{ Factorial points } & 1 & -1 & -1 & -1 & -1 & 37.5 & 37.5 & 37.5 & 37.5 \\
\hline & 2 & +1 & -1 & -1 & -1 & 112.5 & 37.5 & 37.5 & 37.5 \\
\hline & 3 & -1 & +1 & -1 & -1 & 37.5 & 112.5 & 37.5 & 37.5 \\
\hline & 4 & +1 & +1 & -1 & -1 & 112.5 & 112.5 & 37.5 & 37.5 \\
\hline & 5 & -1 & -1 & +1 & -1 & 37.5 & 37.5 & 112.5 & 37.5 \\
\hline & 6 & +1 & -1 & +1 & -1 & 112.5 & 37.5 & 112.5 & 37.5 \\
\hline & 7 & -1 & +1 & +1 & -1 & 37.5 & 112.5 & 112.5 & 37.5 \\
\hline & 8 & +1 & +1 & +1 & -1 & 112.5 & 112.5 & 112.5 & 37.5 \\
\hline & 9 & -1 & -1 & -1 & +1 & 37.5 & 37.5 & 37.5 & 112.5 \\
\hline & 10 & +1 & -1 & -1 & +1 & 112.5 & 37.5 & 37.5 & 112.5 \\
\hline & 11 & -1 & +1 & -1 & +1 & 37.5 & 112.5 & 37.5 & 112.5 \\
\hline & 12 & +1 & +1 & -1 & +1 & 112.5 & 112.5 & 37.5 & 112.5 \\
\hline & 13 & -1 & -1 & +1 & +1 & 37.5 & 37.5 & 112.5 & 112.5 \\
\hline & 14 & +1 & -1 & +1 & +1 & 112.5 & 37.5 & 112.5 & 112.5 \\
\hline & 15 & -1 & +1 & +1 & +1 & 37.5 & 112.5 & 112.5 & 112.5 \\
\hline & 16 & +1 & +1 & +1 & +1 & 112.5 & 112.5 & 112.5 & 112.5 \\
\hline \multirow[t]{6}{*}{ Centre point } & 17 & 0 & 0 & 0 & 0 & 75 & 75 & 75 & 75 \\
\hline & 18 & 0 & 0 & 0 & 0 & 75 & 75 & 75 & 75 \\
\hline & 19 & 0 & 0 & 0 & 0 & 75 & 75 & 75 & 75 \\
\hline & 20 & 0 & 0 & 0 & 0 & 75 & 75 & 75 & 75 \\
\hline & 21 & 0 & 0 & 0 & 0 & 75 & 75 & 75 & 75 \\
\hline & 22 & 0 & 0 & 0 & 0 & 75 & 75 & 75 & 75 \\
\hline \multirow[t]{8}{*}{ Axial points } & 23 & -2 & 0 & 0 & 0 & 0 & 75 & 75 & 75 \\
\hline & 24 & +2 & 0 & 0 & 0 & 150 & 75 & 75 & 75 \\
\hline & 25 & 0 & -2 & 0 & 0 & 75 & 0 & 75 & 75 \\
\hline & 26 & 0 & +2 & 0 & 0 & 75 & 150 & 75 & 75 \\
\hline & 27 & 0 & 0 & -2 & 0 & 75 & 75 & 0 & 75 \\
\hline & 28 & 0 & 0 & +2 & 0 & 75 & 75 & 150 & 75 \\
\hline & 29 & 0 & 0 & 0 & -2 & 75 & 75 & 75 & 0 \\
\hline & 30 & 0 & 0 & 0 & +2 & 75 & 75 & 75 & 150 \\
\hline
\end{tabular}

using CYA106F (5'-TAACACATGCAAGTCGAA-3') and $235^{\prime} \mathrm{R} \quad\left(5^{\prime}\right.$-TCTGTGTGCCTAGGTATCCACCGTT-3') primer pairs (Ballot et al. 2004). PCR was performed according to Ballot et al. (2004). The obtained PCR products were purified using an AccuPrep ${ }^{\mathrm{R}}$ PCR Purification Kit (Bionner, Korea) and sequenced with an ABI Prism ${ }^{\mathrm{TM}} 310$ Genetic Analyzer (Applied Biosystems), using the following primers: CYA106F, 23S5'R, F3 (5'-GTGTAGCGGTGAAA TGCGTAGA-3'), 683R (5' -TCTACGCATTTCACCGCTA CAC-3'), R4R (5'-TACGGCTACCTTGTTACGAC-3'), and $16 \mathrm{~S} 3^{\prime} \mathrm{F}\left(5^{\prime}\right.$-TGTGGCTGGATCACCTCCTT-3'). Nucleotide sequence edition and analysis were performed using ChromasPRO Version 1.49, and DNASTAR (DNASTAR, Inc., UK), BLASTN (Altschul et al. 1997) and FASTA (Pearson and Lipman 1988).
Extraction and quantification of fatty acids

Lipid extraction was done according to Bligh and Dyer (1959). The derivatization of fatty acids was done according to Sato and Murata (1988) and analysed using a Hewlett-Packard (series G1800B) gas chromatograph-mass spectrometer (GC-MS) equipped with Omegawax TM250 fused-silica column (Supelco) of $30 \mathrm{~m} \times 0.25 \mathrm{~mm}$ external diameter $\times 0.25$ um internal diameter. The fatty acids found in the samples were identified by comparing the mass spectra with those contained in the NIST98, NBS75K mass spectral library and in the database created with 28 fatty acid methyl ester standards (Sigma Chemical Company). Additionally, the identification of the fatty acids was confirmed by comparing the retention time of the samples 
Fig. 1 Light micrographs (phase contrast) of Lefevre strain cultures at different steps of the purification strategy; a colonies of Microcystis sp. present after the washing step; b Chroococcus sp. present after $\mathrm{pH}$ treatment for $92 \mathrm{~h}$; in this case, if Lefevre strain survived, its population was significantly smaller than Chroococcus sp. c bacteria present after $\mathrm{pH}$ treatment for $72 \mathrm{~h}$; in this case the population of Lefevre strain was significantly larger than Chroococcus sp., which did not appear under this magnification, indicating that its concentration was low; $\mathbf{d}$ Axenic culture of Lefevre strain after antibiotic treatment and serial dilution. Scale bar $15 \mu \mathrm{m}$
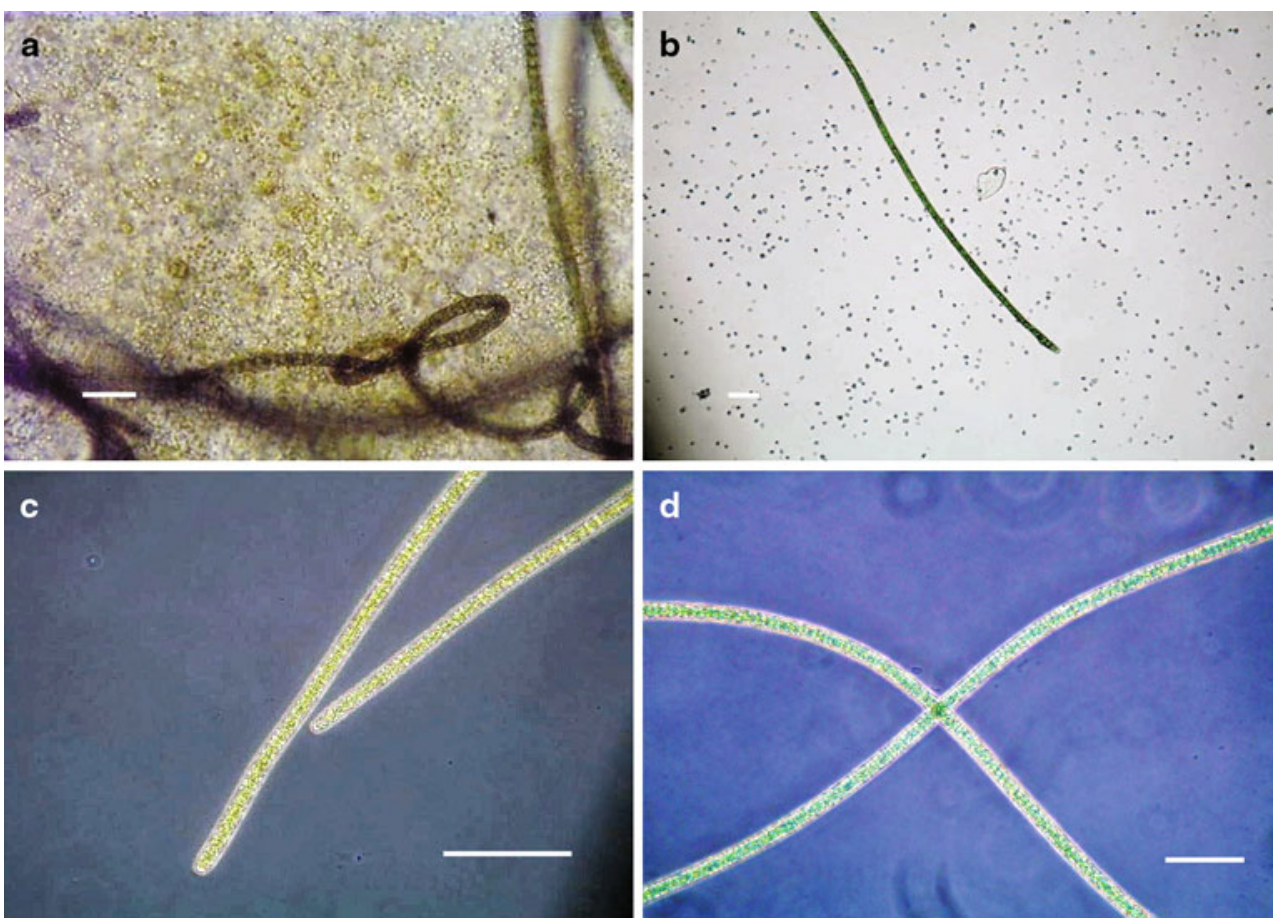

with those registered for a commercial mixture of polyunsaturated fatty acid methyl esters (Sigma). The fatty acid content was calculated and expressed as percent of total fatty acids. The content (\% total fatty acids) of palmitic, linoleic, and $\gamma$-linolenic acids were coded with the same criteria used by Mühling et al. (2006) for this "character state". That is, a value " 0 " was assigned when palmitic, linoleic or $\gamma$-linolenic content was $\leq 46 \%, \leq 17 \%$, or $\leq 26 \%$, respectively, or a value " 1 " otherwize; this with the purpose of comparing with Mühling's results.

\section{Results and discussion}

In order to design a strategy for purification, we performed a preliminary microscopic study of microorganisms present in Arthrospira sp. ('platensis') cultures maintained in our laboratory. A low proportion of ciliated protozoan populations, high proportion of bacteria, and two additional cyanobacteria (taxonomically identified as Microcystis sp. and Chroococcus sp.) were observed (Fig. 1a). Based on these observations, we designed a strategy for selective elimination of the contaminant bacteria, cyanobacteria and protozoan. To obtain the axenic culture, the first step planned was washing, since that significantly reduces the population of microorganisms smaller than Arthrospira filaments. The second step was $\mathrm{pH}$-treatment to eliminate protozoan and Microcystis sp. (time and $\mathrm{pH}$ were used to optimize this step, see Table 2). Arthrospira (Spirulina) spp. can live in extremely alkaline environments, up to approximately pH 12 (Ciferri 1983), while most microorganisms cannot resist these conditions (due to osmotic shock and cell lysis); consequently, a $\mathrm{pH}$ treatment was an obvious choice. The desired $\mathrm{pH}$ levels were tuned using potassium hydroxide $(\mathrm{KOH})$ due to its strong inhibition of Microcystis sp. growth (Parker et al. 1997). The treatment

Table 2 Results of pH treatments: microorganisms present or absent in Arthrospira sp. ('platensis') cultures after each combination of time and $\mathrm{pH}$

\begin{tabular}{|c|c|c|c|c|c|c|c|c|c|c|c|c|c|c|c|c|c|c|c|c|}
\hline \multirow[t]{3}{*}{ Time (h) } & \multicolumn{20}{|c|}{ pH levels } \\
\hline & \multicolumn{5}{|c|}{10} & \multicolumn{5}{|l|}{11} & \multicolumn{5}{|l|}{12} & \multicolumn{5}{|l|}{13} \\
\hline & A & $\mathrm{P}$ & M & $\mathrm{C}$ & B & A & $\mathrm{P}$ & M & $\mathrm{C}$ & $\mathrm{B}$ & A & $\mathrm{P}$ & M & $\mathrm{C}$ & $\mathrm{B}$ & A & $\mathrm{P}$ & M & $\mathrm{C}$ & B \\
\hline 48 & + & + & + & + & + & + & + & + & + & + & + & - & + & + & + & - & - & - & + & + \\
\hline 72 & + & + & + & + & + & + & - & + & + & + & + & - & - & + & + & - & - & - & - & - \\
\hline 96 & + & - & + & + & + & + & - & + & + & + & + & - & - & + & + & - & - & - & - & - \\
\hline
\end{tabular}

Arthrospira sp. ('platensis') (A); protozoan (P); Microcystis sp. (M); Chroococcus sp. (C); bacteria (B); present (+); absent (-) 
at $\mathrm{pH} 12$ for $72 \mathrm{~h}$ was found to be the most effective because no protozoa or Microcystis sp. were observed in the treated culture (Fig. 1c). Even though, pH 12 for $96 \mathrm{~h}$ also eliminated protozoa and Microcystis sp., few filaments of Arthrospira were observed and its population was significantly smaller than Chroococcus sp. (Fig. 1b). Lower $\mathrm{pH}$ levels (10 or 11) or treatments shorter than $72 \mathrm{~h}$ did not eliminate contamination due to these microorganisms, and a higher $\mathrm{pH}$ of 13 killed Lefevre strain (see Table 2). Based on these findings, we decided to apply a pool of antibiotics that were selected to eliminate bacteria that survived the $\mathrm{pH}$ treatment. Furthermore, to obtain the optimal concentration of the selected antibiotics, a statistical experiment (CCRD) was designed and conducted. The chosen antibiotics (ampicillin, cefoxitin, penicillin $\mathrm{G}$ and meropenem) are potent $\beta$-lactams that inhibit cell wall synthesis of bacteria, especially in Gram-positive organisms. The $\beta$-lactam ring strongly binds to transpeptidases PBPs (penicillin-binding proteins), inhibiting the final crosslinking reactions of peptidoglycan synthesis during cell wall formation (Madigan et al. 1997). The antibiotic-PBP complex also stimulates autolysin liberation that digests the existing cell wall (Donowitz and Mandell 1988).

The results of the CCRD experiment showed that treatments 6, 8, 13, 14 and 19 allowed the growth of Arthrospira (see Fig. 2), but only treatments 8 and 14 produced bacteria-free cultures. For all the antibiotic treatments, Arthrospira showed a lag phase of approximately 16 days, indicating that the antibiotics also affected the cyanobacterium. This behaviour was expected, because Arthrospira also synthesizes a peptidoglycan layer. However, the effect of these antibiotics on this cyanobacterium was expected to be less pronounced than on bacteria, given that Arthrospira has a multilayer cell wall (similar to Gram-negative bacteria surrounded by a mucilaginous cover) and longer generational time than bacteria.

Lack of variance homogeneity in the data was related to the relatively small number of treatments that presented bacterial or Arthrospira growth. Therefore, a raw data transformation was applied for the number of bacteria to fulfil the assumption of variance homogeneity. The variance analysis (ANOVA) for the antibiotic effect on bacteria indicated that the model used is significant ( $p$-value 0.0482 ). It also showed that cefoxitin is the antibiotic that presented a significant effect on bacteria ( $p$-value 0.00062). Based on this analysis, the regression equation obtained was (Eq. 2)

$$
\begin{aligned}
& \frac{1}{\sqrt{[10](\text { number of bacteria }+11,400)^{2,36}}} \\
& =2.08 \times 10^{-11}-1.94 \times 10^{-19} X_{1}+1.26 \times 10^{-13} X_{2} \\
& \quad-6.29 \times 10^{-14} X_{3}+1.94 \times 10^{-19} X_{4}
\end{aligned}
$$

where, $X_{1}, X_{2}, X_{3}$ and $X_{4}$ are the independent variables corresponding to ampicillin, cefoxitin, penicillin $\mathrm{G}$ and meropenem concentrations, respectively. The equation shows that the coefficient for cefoxitin $\left(X_{2}\right)$ is positive $\left(+1.26 \times 10^{-13}\right)$. Note that the data transformation is an inverse function; therefore, the real effect of cefoxitin on bacterial growth is negative. These results are in agreement with the following facts: (1) bacteria present in Arthrospira spp. cultures are diverse but have a prevalence of Gramnegative rods (Ogawa and Terui 1970); (2) Cefoxitin is an extended-spectrum antibiotic with major effects on Gram-
Fig. 2 Number of bacteria (c.f.u.) and Lefevre strain filaments after the antibiotic treatments $\left(2^{4}\right.$ Factorial CCRD experiment)

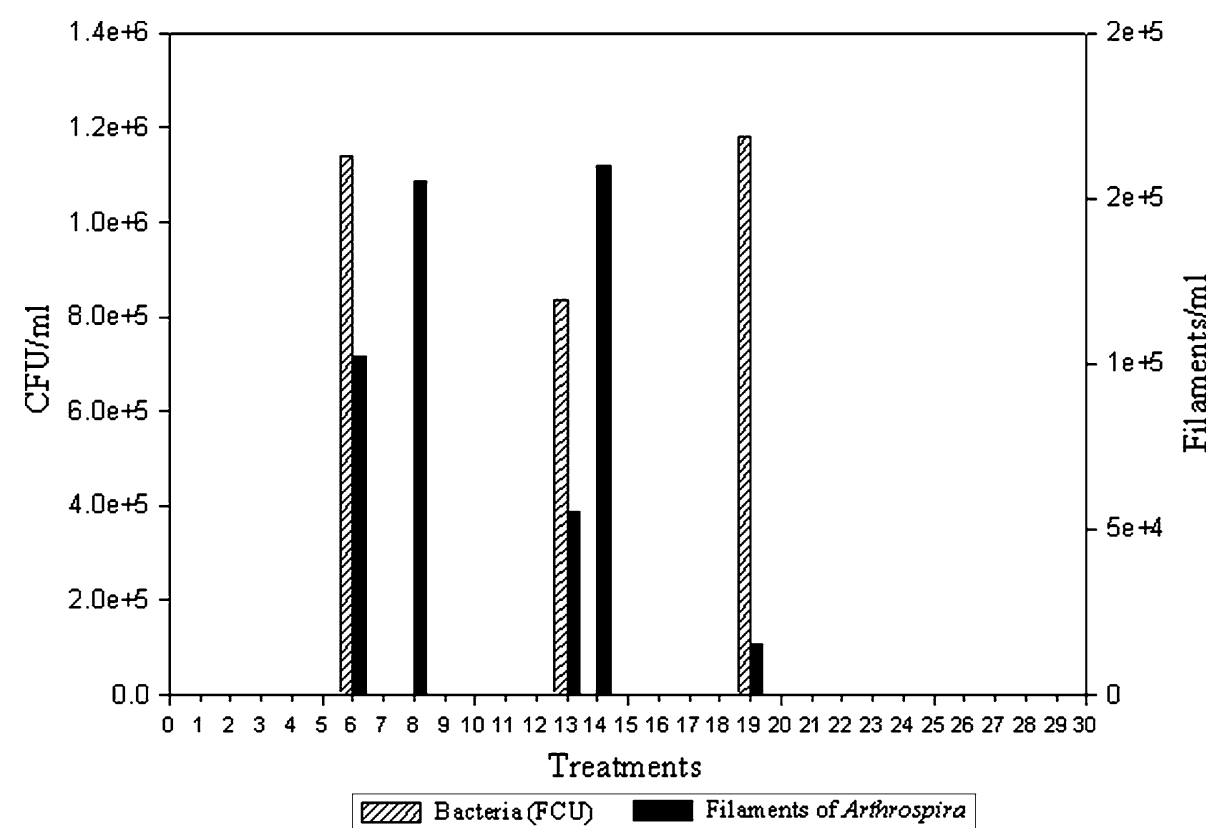


negative bacteria (Page 1992); (3) Even though cefoxitin has the same action mechanism as penicillin, its different molecular structure makes it more resistant to $\beta$-lactamases and substances that destroy the $\beta$-lactam ring, extending its activity without becoming easily degraded.

The results of this experiment indicate that the optimal concentration of each antibiotic (in the pool) to obtain bacteria-free cultures of Arthrospira are: ampicillin $61.6 \mu \mathrm{g} / \mathrm{ml}$, penicillin $85.8 \mu \mathrm{g} / \mathrm{ml}$, cefoxitin $76.9 \mu \mathrm{g} / \mathrm{ml}$, and meropenem $38.9 \mu \mathrm{g} / \mathrm{ml}$. In order to test this set of optimal concentrations, the pool was applied to Arthrospira cultures, in triplicate, previously $\mathrm{pH}$-treated $(\mathrm{pH} 12$ for $72 \mathrm{~h}$ ), and bacteria-free cultures were obtained for all the replicates. However, after the optimal antibiotic treatment was applied, a very low proportion of the cyanobacterium Chroococcus sp. was present in the cultures. This cyanobacterium has a thick sheath and intrinsic characteristics that makes it resistant to adverse conditions such as desiccation, high temperature, high and low $\mathrm{pH}$ levels and chemicals. Therefore, serial dilutions were used to eliminate it. The dilution of 1:10,000 was effective to discard Chroococcus sp., and axenic cultures of Arthrospira sp. ('platensis') Lefevre 1963/M-132-1 strain were successfully obtained (Fig. 1d).

Once the axenic culture was obtained, we proceeded to carry out a molecular identification. The size of the DNA sequence obtained was 1,948 bp (GenBank Accession number FJ798612.1) and was compared against the GenBank (NCBI) database. The results showed that A. platensis (GenBank accession numbers: EF432320; DQ279770; DQ279768; EF432312; DQ279767; DQ279769; EF432316) presented the highest sequence similarity (99\% of identity) with Lefevre 1963/M-132-1 strain. Through this analysis, it was found that indeed the purified strain belongs to the genus Arthrospira (which could be considered sufficient for the purposes of this work); however, doubts about its exact identity remain.

In contradiction to the results on molecular identification, the content of fatty acids (palmitic, linoleic and $\gamma$-linolenic) was $48.7,21.2,13.9 \%$, respectively, or $1,1,0$ (data coded), which are identical to those obtained by Mühling et al. (2006) (see strain D873, strain characters 23, 24 and 25) and would place our strain in the group of $A$. maxima, A. fusiformis and A. indica. This corresponds to phenotypic cluster I, according to Mühling's notation or genetic cluster I according to Scheldeman et al. (1999). This result suggests that Lefevre 1963/M-132-1 strain could be more closely related to A. maxima.

It remains to ask why this contradiction arises and what the possible explanation is. An insight into a potential explanation for this finding can be found in the words of Mühling et al. (2006):
On the basis of a comparison of the trichome morphology of these isolates with descriptions in the literature for the species name used in the original culture collection, it seems likely that several had been assigned the wrong name.

It is important to note that the results of Mühling et al. (2006) and Scheldeman et al. (1999) refer to concrete measurements that are not subject to interpretation, hence the almost perfect match. On the other hand, when working with classical taxonomy, other variables, such as the skills and experience of the taxonomist, the interpretation of the original descriptions, etc., come into play. When a sample is misclassified we run the risk that the error is transmitted to reach databases such as the GenBank, where sequences are deposited under a wrong name, and thereby the identification based only on comparing against sequences deposited in this database can lead to errors. As we have demonstrated here, we should assume this task with as many tools and methods available.

As for the true identity of the strain Lefevre 1963/M132-1, we consider that it is A. maxima, which is clearly evidenced by its clustering near Arthrospira sp. ('platensis') Laporte 1963/M 132-2b in the dendrogram built by Scheldeman et al. (1999). This strain was also reclassified as A. maxima (in the CCALA) after the publication of that work.

Acknowledgments This research was supported by BID-FONACIT Project No. 200600537. We thank the Genomic Polymorphism Unit from Health Direction of IDEA Foundation (Fundación IDEA), José Alexander Parra, Armando Sena, Bertha Arredondo-Vega, Diego Rodriguez, Jaromir Lukavsky, Jirí Komárek, José Luis Ramirez, and two anonymous reviewers for asking for useful clarification, and whose comments and suggestions allowed us to improve this manuscript.

Open Access This article is distributed under the terms of the Creative Commons Attribution Noncommercial License which permits any noncommercial use, distribution, and reproduction in any medium, provided the original author(s) and source are credited.

\section{References}

Aiba S, Ogawa T (1977) Assessment of growth yield of a blue-green alga: Spirulina platensis, in axenic and continuous culture. J Gen Microbiol 102:179-182

Altschul SF, Madden TL, Schaffer AA, Zhang J, Zhang Z, Miller W, Lipman DJ (1997) Gapped BLAST and PSI-BLAST: a new generation of protein database search programs. Nucleic Acids Res 25:3389-3402

Andersen RA (2005) Algal culturing techniques. Andersen RA (eds), Elsevier, pp 117-132

Ballot A, Dadheech P, Krienitz L (2004) Phylogenetic relationship of Arthrospira, Phormidium and Spirulina strains from Kenia and Indian waterbodies. Algol Stud 113:37-56

Baurain D, Renquin L, Grubisic S, Scheldeman P, Belay A, Wilmotte A (2002) Remarkable conservation of internally transcribed 
spacer sequences of Arthrospira ("Spirulina") (Cyanophyceae, Cyanobacteria) strains from four continents and of recent and 30-year-old dried samples from Africa. J Phycol 38:384-393

Bligh EG, Dyer WJ (1959). A rapid method for total lipid extraction and purification. Can J Biochem Physiol 37:911-917

Boyer SL, Flechtner VR, Johansen JR (2001) Is the 16S-23S rRNA internal transcribed spacer region a good tool for use in molecular systematics and population genetics? A case study in cyanobateria. Mol Biol Evol 18:1057-1069

Carmichael WW, Gorham PR (1974) An improve method for obtaining axenic clones planctonic blue green algae. J Phycol 10:238-240

Castenholz RW (1970) Laboratory culture of thermophilic cyanophytes. Schweiz Z Hydrol 32:538-551

Ciferri O (1983) Spirulina, the edible microorganism. Microbiol Rev 47:551-578

Ciferri O, Tiboni O (1985) The biochemistry and industrial potential of Spirulina. Annu Rev Microbiol 39:503-526

Cohen Z, Vonshak A (1991) Fatty acid composition of spirulina and spirulina-like cyanobacteria in relation to their chemotaxonomy. Phytochemistry 30:205-206

Cohen Z, Margheri MC, Tomaselli L (1995) Chemotaxonomy of cyanobacteria. Phytochemistry 40(4):1155-1158

Donowitz GR, Mandell GL (1988) Beta-lactam antibiotics. N Engl J Med 318:419-426

Duerr EO, Edralin MR, Price NM (1997) Facilities requirements and procedures for the laboratory and outdoor raceway culture of Spirulina spp. J Mar Biotechnol 5:1

Ferris MJ, Hirsch CF (1991) Methods for isolation and purification of cyanobacteria. Appl Environ Microbiol 57:1448-1452

Gang-Guk C, Myong-Sook B, Chi-Yong A, Hee-Mock O (2007) Induction of axenic culture of Arthrospira (Spirulina) platensis based on antibiotic sensitivity of contaminating bacteria. Biotecnol Lett 30:87-92

Hindak F (1985) Morphology of trichomes in Spirulina fusiformis Voronichin from Lake Bogoria, Kenya, Arch Hydrobiol Suppl. 71, Algol Stud, 38/39, 201

Kim JS, Park YH, Yoon BD, Oh HM (1999) Establishment of axenic cultures of Anabaena flos-aquae and Aphanotheca nidulans (cyanobacteria) by lysozyme treatment. J Phycol 35:865-869

Komárek J (2000) Problems in cyanobacterial taxonomy: implication for most common toxin producing species. In: Istituto Superiore di Sanità Workshop. Freshwater harmful algal blooms: health risk and control management. Istituto Superiore di Sanità. Rome, 17 October 2000. In: Proceedings edited by Serena Melchiorre, Emanuela Viaggiu and Milena Bruno 2002, 103 p. Rapporti ISTISAN 02/9 (in Italian and English)

Komárek J, Anagnostidis K (2005) Cyanoprokaryota-2. Teil/2nd Part: Oscillatoriales. In: Büdel B, Krienitz L, Gärtner G, Schagerl M (eds) Süsswasserflora von Mitteleuropa 19/2, Elsevier/Spektrum, Heidelberg, p 759

Komárek J, Lund JWG (1990) What is 'Spirulina platensis' in fact? Arch Hydrobiol Suppl 85 Algol Stud 58:1

Madigan M, Martinko J, Parker J (1997) Brock biología de los microorganismos. Décima ed. Prentice-Hall Iberia. Madrid, p 1096

Manen JF, Falque J (2002) The cpcB-cpcA locus as a tool for the genetic characterization of the genus Arthrospira (Cyanobacteria): evidence for horizontal transfer. Int J Syst Evol Microbiol $52: 861-867$

Montgomery DC (1997) Design and analysis of experiments, 3rd edn. Wiley, New York, p 680

Mosulishvili LM, Kirkesali EI, Belokolbylsky AI, Khizanishvili AI, Frontasyeva MV, Pavlov SS, Gundorina SF (2002) Experimental substantiation of the possibility of developing selenium and iodine containing pharmaceuticals based on blu-green algae Spirulina platensis. J Pharm Biomed Anal 30:87-97
Mühling M, Belay A, Whitton BA (2005) Variation in fatty acid composition of Arthrospira (Spirulina) strains. J Appl Phycol 17:137-146

Mühling M, Sommerfield PJ, Harris N, Belay A, Whitton BA (2006) Phenotypic analysis of Arthrospira (Spirulina) strains (Cyanobacteria). Phycologia 45:148-157

Myers RH, Montgomery DC (2002) Response surface methodology: process and product optimization using designed experiments, 2nd edn. Wiley, London

Nola MC, Smaglinski ST, Smith GD (1992) Methods involving light variation for isolation of cyanobacteria: characterization of isolates from Central Australia. Appl Environ Microbiol 58: $3561-3566$

Ogawa T, Terui G (1970) Studies on the growth of Spirulina platensis. (I) On the pure culture of Spirulina platensis. Ferment Tecnnol 48:361-367

Page MI (1992) The chemistry of $\beta$-lactams. Chapman and Hall, New York

Parker DL, Kumar HD, Rai LC, Sing JB (1997) Potassium salts inhibit growth of the cyanobacteria Microcystis spp. in pond water and defined media: implications for control of microcystin-producing aquatic blooms. Appl Environ Microbiol 63: 2324-2329

Pearson W, Lipman D (1988) Improved tools for biological sequence comparison. Proc Natl Acad Sci 85:2444-2448

Rippka R (1988) Isolation and purification of cyanobacteria. Meth Enzymol 167:3-27

Sato N, Murata N (1988) Membrane lipids. Meth Enzymol 167: 251-259

Scheldeman P, Baurain D, Bouhy R, Scott M, Muhling M, Whitton BA, Belay A, Wilmotte A (1999) Arthrospira ('Spirulina') strains from four continents are resolved into only two clusters, based on amplified ribosomal DNA restriction analysis of the internally transcribed spacer. FEMS Microbiol Lett 172:213-222

Suresh PT, Raman MK, Ramamurthy V (1994) Obtaining axenic culture of filamentous cyanobacterium. Biotechniques 6: 216-217

Tomaselli L (1997) Morphology, ultrastructure and taxonomy of Arthrospira (Spirulina) maxima and Arthrospira (Spirulina) platensis In: Vonshak A (eds) Spirulina platensis (Arthrospira): physiology, cell-biology and biotechnology. Taylor \& Francis, London, UK, pp 1-19

Vaara T, Vaara M, Niemela S (1979) Two improved methods for obtaining axenic cultures of cyanobacteria. Appl Environ Microbiol 38:1011-1014

Viti C, Ventura S, Lotti F, Capolino E, Tomaselli L, Giovannetti L (1997) Genotypic diversity and typing of cyanobacterial strains of the genus Arthrospira by very sensitive total DNA restriction profile analysis. Res Microbiol 148:605-611

Vonshak A (1997) Spirulina: growth, physiology and biochemistry. In: Vonshak A (eds) Spirulina platensis (Arthrospira): physiology, cell-biology and biotechnology. Taylor \& Francis, London, UK, pp 79-99

Wang ZP, Zhao Y (2005) Morphological reversion of Spirulina (Arthrospira) platensis (Cyanophyta): from linear to helical. J Phycol 41:622-628

Wang HB, Zhu HG (1996) Promoting activity of microcystin extracted from warerblooms in SHE cell transformation assay. Biomed Environ Sci 9:46-51

Wieringa KT (1968) A new method for obtaining bacteria-free cultures of blue-green algae. Antonie van Leeuwenhoek J Microbiol Serol 34:54-56

Wu X, Zarka A, Boussiba S (2002) A simplified protocol for preparing DNA from filamentous cianobacteria. Plant Mol Biol $18: 385-392$ 